\title{
Cluster partitioning of heterogeneous multi-agent systems *
}

\author{
Mattia Mattioni ${ }^{\text {a }}$, Salvatore Monaco ${ }^{\text {a }}$

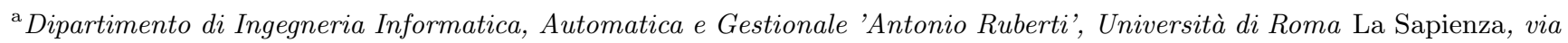 \\ Ariosto 25, 00185 Roma, Italy.
}

\begin{abstract}
This paper investigates the collective behaviors induced by the network interconnection of heterogeneous input-affine singleinput nonlinear systems through a constant but general directed graph. In this sense, we prove that the dynamics of all agents cluster into as many subgroups as the number of cells of the almost equitable partition induced by the communication graph. All agents belonging to the same cell are equally influenced by a new mean-field dynamics which is paradigmatic of the network. The case of a network of pendula illustrates the results through simulations.
\end{abstract}

Key words: Multiconsensus; Multi-agent systems; Linear/nonlinear models.

\section{Introduction}

Networked systems are nowadays considered a bridging paradigm among several disciplines spanning, among many others, from physics to engineering, psychology to medicine, biology to computer science. As typical in control theory (Isidori (2017)), we refer to a network (or multi-agent) system as composed of several dynamical units (agents) interconnected through a communication graph: each node of the communication graph uniquely corresponds to one dynamical unit whereas edges model the exchange of information among agents. As a consequence, even for simple agents and with no issue in the network interconnection (e.g., time-delays), the network behavior is described by a complex dynamical system. In these regards, several works have been devoted to providing methodological understanding on the collective behavior induced by the network interaction through the graph. Such a behavior might be declined into different scenarios such as flocking, randez-vous, formation control and swarming allowing to describe a huge number of control problems in a unifying framework. Most of these can be generally lead to the possibility of driving all systems composing the network toward a consensus behavior that might be global for all individuals or common only to some clusters uniquely identified by the

\footnotetext{
‡ Partially supported by Università degli Studi di Roma La Sapienza through the Progetti di Ateneo 2019 (Piccoli progetti RP11816436325B63).
}

Email addresses: mattia.mattioni@uniroma1.it

(Mattia Mattioni), salvatore.monaco@uniroma1.it (Salvatore Monaco). graph (e.g., Jadbabaie et al. (2003); Olfati-Saber et al. (2007); Aeyels and De Smet (2010); Ren and Cao (2010); Chen et al. (2011); Egerstedt et al. (2012); Wang and Lu (2019); Gambuzza and Frasca (2020); Mattioni (2020); Cristofaro and Mattioni (2021)).

In case of integrator dynamics, the consensus of the network is dictated by the properties of the underlying graph. Starting from this and motivated by practical applications, several ad-hoc studies have been carried out for larger classes of phenomena and systems such as chaotic oscillators and circuits, mobile and autonomous robots, power systems, cyber security or opinion dynamics by assuming particular structures of the graph underlying the interconnection and, possibly, under sampling, delays or disturbance actions on the nodes (e.g., Moreau (2005); Sun and Wang (2009); Arenas et al. (2008); Dimarogonas et al. (2012); Cui et al. (2012); Chen et al. (2013); Pasqualetti et al. (2013); Zhan and Li (2013); Wen et al. (2013); Battilotti and Califano (2019); Pietrabissa and Suraci (2017); DeLellis et al. (2018); Trumpf and Trentelman (2018)). Those works, focused on single consensus, suggest that the evolutions of each unit are strongly affected by the network topology, the type and strength of the interconnection and the dynamics of each unit. However, general results allowing to understand the simultaneous influence of these three features of the network dynamics are unavailable.

In Panteley and Loría (2017) the case of heterogeneous (input-output) feedback linearizable systems under diffusing coupling and a strongly connected graph has been investigated. In particular, it is explicitly revealed that the agents' interconnection generates a network behavior (the so-called mean-field dynamics) which depends, 
through the underlying graph, on a suitable combination of each unit's dynamics. When agents evolve identically, it reduces to the so-called emergent dynamics. Those new dynamics are of paramount importance as they offer a unifying framework for dealing with several problems related to multi-agent systems. Further developments of this approach have been proposed in Lee and Shim (2020) for classes of heterogeneous multi-agent systems also embedding distributed control design. At the same time, in Monaco and Ricciardi Celsi (2019) the multiple consensus behaviors and their clustered distribution in a network of simple integrators have been precisely characterized for a general digraph exploiting the notion of almost equitable partition (Cardoso et al. (2007); Caughman and Veerman (2006)).

The aforementioned results motivate the present work whose contribution stands in providing an exact characterization of the behaviors induced by the topology over a network of heterogeneous nonlinear systems under diffusely coupling (through their outputs). No assumptions on the graph structure nor on the agent dynamics are set. Namely, it is proved that, as the intuition suggests, exploiting the properties inferred in Monaco and Ricciardi Celsi (2019), the network topology induces as many mean-field dynamics as the number of reaches of the communication graphs; each mean-field dynamics uniquely affects a suitable cluster of agents. In addition, the common component to all reaches splits into further sub-groups, each of which is driven by a suitable combination of the mean-field dynamics associated to the reaches. In this sense, the emergent and mean-field dynamics are composed of as many independent units as the number of exclusive reaches. The clusters arising in the network (and in particular within the common) are uniquely associated to the almost equitable partition associated to the communication graph $\mathcal{G}$, as in the case of simple scalar integrators. The results here developed are reminiscent of the ones in Frasca et al. (2018) dealing with to multi-clustered consensus of chaotic oscillators and circuits and the ones in Liu et al. (2012); Xiao and Wang (2006) for the case of LTI homogeneous dynamics.

The rest of the paper is organized as follows. In Section 2, preliminaries on graphs and Laplacian matrices are given and the problem is settled. The main results are in Section 3 by providing the structure of the network through the emergent and mean-field dynamics and the corresponding behavior they induce through the synchronization errors. The example of a network of gravity pendula is illustrated in Section 4 while Section 5 concludes the paper.

Notations. The sets $\mathbb{C}, \mathbb{R}$ and $\mathbb{N}$ denote the set of complex, real and natural numbers including 0 respectively. $\mathbb{C}^{+}$and $\mathbb{C}^{-}$denote the right and left half of the complex plane respectively. The symbols " $>0$ " and " $<0$ " denote positive and negative definite functions whereas $\succ$ and $\prec(\succeq$ and $\preceq)$ positive and negative (semi) definite matrices. $I_{n}$ denotes the identity matrix of dimension $n \geq 1$ whereas $\mathbf{0}$ is the zero-matrix of suitable dimensions. For a square matrix $A \in \mathbb{R}^{n \times n}, \sigma(A) \subset \mathbb{C}$ denotes its spectrum. Given $m$ column vectors $g_{j} \in \mathbb{R}^{n}$ with $j=1, \ldots, m$ we denote by $\operatorname{diag}\left(g_{1}, \ldots, g_{m}\right) \in \mathbb{R}^{m n \times m}$ the block-diagonal matrix with $g_{j}$ in the main diagonal whereas $\operatorname{col}\left(g_{1}, \ldots, g_{m}\right)=\left(g_{1}^{\top} \ldots g_{m}^{\top}\right)^{\top} \in \mathbb{R}^{n m}$. Given to matrices $A \in \mathbb{R}^{n_{1} \times n_{2}}$ and $B \in \mathbb{R}^{m_{1} \times m_{2}}$, the Kronecker product is denoted by $A \otimes B$. For a given set $\mathcal{S}$, $|\mathcal{S}|$ denotes its cardinality. If $(\mathcal{X}, d)$ is a metric space, $\Gamma \subset \mathcal{X}$ and $x \in \mathcal{X}$, then $\|x\|_{\Gamma}=\inf _{y \in \Gamma} d(x, y)$ defines the point-to-set distance of $x$ to $\Gamma$.

\section{Preliminaries and problem statement}

In this section we recall, employing for the usefulness of the reader the same notations too, the result achieved in Monaco and Ricciardi Celsi (2019) with reference to the multi consensus of a network of integrators.

\subsection{Recalls on graph theory}

Consider a digraph (that is an unweighted directed graph) $\mathcal{G}=\{\mathcal{V}, \mathcal{E}\}$ with $\mathcal{V}$ being the set of vertices with cardinality $|\mathcal{V}|=N$ and $\mathcal{E} \subseteq \mathcal{V} \times \mathcal{V}$ being the set of edges (i.e., the set of ordered pairs of node). For all pairs of distinct notes $\nu_{i}, \nu_{j} \in \mathcal{V}$ then $\left(\nu_{i}, \nu_{j}\right) \in \mathcal{E}$ if there exists an edge from $\nu_{i}$ to $\nu_{j}$ or, equivalently, $\nu_{i}$ is a neighbour of $\nu_{j}$ for all $i \neq j=1, \ldots, N$. For all pairs of distinct nodes $\nu, \mu \in \mathcal{V}$, a directed path from $\nu$ to $\mu$ is defined as $\nu \leadsto \mu=\left\{\left(\nu_{r}, \nu_{r+1}\right) \in \mathcal{E}\right.$ s.t. $\cup_{r=0}^{\ell-1}\left(\nu_{r}, \nu_{r+1}\right) \subseteq$ $\mathcal{E}$ with $\nu_{0}=\nu, \nu_{\ell}=\mu$ and $\left.\ell>0\right\} . \mathcal{G}$ is said to be: weakly connected if its undirected version is connected and there is no unreachable node (that is there exists a path between all pairs of nodes); rooted if it is weakly connected and contains at least one rooted out-branching; strongly connected if there always exists a directed path between every pair of nodes and there is no unreachable node. The reachable set from a node $\nu \in \mathcal{V}$ is defined as $R(\nu):=\{\nu\} \cup\{\mu \in \mathcal{V}$ s.t. $\nu \leadsto \mu\}$. A set $\mathcal{R}$ is called a reach if it is a maximal reachable set that $\mathcal{R}=R(\nu)$ for some $\nu \in \mathcal{V}$ and there is no $\mu \in \mathcal{V}$ such that $R(\nu) \subset R(\mu)$. Since $\mathcal{G}$ possesses a finite number of vertices, such maximal sets exist and are uniquely determined by the graph itself. Let $\mathcal{R}_{i}$ with $i=1, \ldots, \mu$ and $\mu \leq N$ denote the (not empty) reaches of the graph $\mathcal{G}$. For each reach $\mathcal{R}_{i}, \mathcal{H}_{i}=\mathcal{R}_{i} / \cup_{j=1, j \neq i}^{\mu} \mathcal{R}_{j}$ with $h_{i}=\left|\mathcal{H}_{i}\right|$ defines the exclusive part while $\mathcal{C}_{i}=\mathcal{R}_{i} / \mathcal{H}_{i}$ is the corresponding common part whose union defines $\mathcal{C}=\cup_{i=1}^{\mu} \mathcal{C}_{i}$ with $\delta=|\mathcal{C}|$.

The set of neighbours associated to $\nu_{i} \in \mathcal{V}$ is defined as $\mathcal{N}_{i}=\left\{\nu \in \mathcal{V} /\left\{\nu_{i}\right\}\right.$ s.t. $\left.\left(\nu, \nu_{i}\right) \in \mathcal{E}\right\}$ with cardinality $d_{i}=\left|\mathcal{N}_{i}\right|$ being also referred to as in-degree of $v_{i}$. Accordingly, the in-degree matrix is defined as $\mathcal{D}=\operatorname{diag}\left(d_{1}, \ldots, d_{N}\right) \in \mathbb{R}^{N \times N}$ whereas the adjacency matrix is $\mathcal{A}=\left\{a_{i j}\right\} \in \mathbb{R}^{N \times N}$ with $a_{i i}=0$ and $a_{i j}=1$ if $\left(\nu_{i}, \nu_{j}\right) \in \mathcal{E}$ and $a_{i j}=0$ otherwise. The Laplacian of $\mathcal{G}$ is $\mathcal{L}=\mathcal{D}-\mathcal{A}$ and possesses one eigenvalue $\lambda=0$ 
with algebraic multiplicity $\mu$ being equal to the number of reaches of $\mathcal{G}$ and all other eigenvalues in the lefthand side of the complex plane (Agaev and Chebotarev (2005); Caughman and Veerman (2006)). As a result, it has been shown in Caughman and Veerman (2006); Monshizadeh et al. (2015); Monaco and Ricciardi Celsi (2019) that, after suitably reordering the graphs nodes, the Laplacian $\mathcal{L}$ always admits the following upper triangular form

$$
\mathcal{L}=\left(\begin{array}{ccccc}
\mathcal{L}_{1} & \mathbf{0} & \ldots & \mathbf{0} & \mathbf{0} \\
\mathbf{0} & \mathcal{L}_{2} & \ldots & \mathbf{0} & \mathbf{0} \\
& & \ddots & & \\
0 & \mathbf{0} & \ldots & \mathcal{L}_{\mu} & \mathbf{0} \\
\mathcal{M}_{1} & \mathcal{M}_{2} & \ldots & \mathcal{M}_{\mu} & \mathcal{M}
\end{array}\right)
$$

with each $\mathcal{L}_{i} \in \mathbb{R}^{h_{i} \times h_{i}}$ being the Laplacian associated to the strongly connected subgraph induced by the exclusive $\mathcal{H}_{i} ; \mathcal{M} \in \mathbb{R}^{\delta \times \delta}$ and $\mathcal{M}_{i} \in \mathbb{R}^{\delta \times h_{i}}$ define, respectively, the internal connections in the common part $\mathcal{C}$ of the digraph and the ones with the exclusive $\mathcal{H}_{i}$. As a consequence, each $\mathcal{L}_{i}$ possesses an eigenvalue in $\lambda=0$ with algebraic multiplicity 1 whereas $\sigma(\mathcal{M}) \subset \mathbb{C}^{+}$. By this structure, it is evident that the eigenspace associated to the zero eigenvalue is hence given by $E=$ $\operatorname{span}\left\{z_{1}, \ldots, z_{\mu}\right\}$ with

$$
z_{1}=\left(\begin{array}{lllll}
\mathbb{1}_{h_{1}}^{\top} & \ldots & \mathbf{0}^{\top} & \gamma^{\top}
\end{array}\right)^{\top}, \ldots, z_{\mu}=\left(\begin{array}{lll}
\mathbf{0}^{\top} & \ldots & \mathbb{1}_{h_{\mu}}^{\top} \gamma^{\mu \top}
\end{array}\right)^{\top}
$$

with $\mathbb{1}_{p}$ being the $p$-dimensional vector whose entries are one, $\sum_{i=1}^{\mu} \gamma^{i}=\mathbb{1}$ and verifying $\mathcal{L}_{i} \mathbb{1}_{h_{i}}=0$ and $\mathcal{M}_{i} \mathbb{1}_{h_{i}}+$ $\mathcal{M} \gamma^{i}=0$ for all $i=1, \ldots, \mu$. Correspondingly, the left eigenvectors associated to the zero eigenvalue of $\mathcal{L}$ read

$$
\tilde{v}_{1}^{\top}=\left(\begin{array}{lllll}
v_{1}^{\top} & \mathbf{0} & \ldots & \mathbf{0} & \mathbf{0}
\end{array}\right), \ldots, \tilde{v}_{\mu}^{\top}=\left(\begin{array}{lllll}
\mathbf{0} & \mathbf{0} & \ldots & v_{\mu}^{\top} & \mathbf{0}
\end{array}\right)
$$

with $v_{i}^{\top}=\left(v_{i}^{1} \ldots v_{i}^{h_{i}}\right)$ verifying for all $i=1, \ldots, \mu$ (denoting $\left.h_{0}=0\right): v_{i}^{\top} \mathcal{L}_{i}=0 ; v_{i}^{\top} \mathbb{1}_{h_{i}}=1$; for each $s=$ $1, \ldots, h_{i}, v_{i}^{s}>0$ if $\nu_{h_{1}+\cdots+h_{i-1}+s} \in \mathcal{H}_{i}$ is a root and $v_{i}^{s}=0$ otherwise. Moreover, if $\mathcal{M}_{i}=\mathbf{0}$ the reach $\mathcal{H}_{i}$ defines a disconnected component of $\mathcal{G}$.

A partition $\pi=\left\{\rho_{1}, \ldots, \rho_{r}\right\}$ of $\mathcal{V}$ is a collection of cells $\rho_{i} \subseteq \mathcal{V}$ verifying $\rho_{i} \cap \rho_{j}=\emptyset$ for all $i \neq j$ and $\cup_{i=1}^{r} \rho_{i}=$ $\mathcal{V}$. A partition $\pi=\left\{\rho_{1}, \ldots, \rho_{r}\right\}$ of $\mathcal{V}$ is said to be an almost equitable partition (AEP, in short) if each node of $\rho_{i}$ has the same number of neighbors in $\rho_{\ell}$, for all $i, \ell \in\{1, \ldots, r\}$ with $i \neq \ell$. More precisely, for a node $\nu_{i} \in \mathcal{V}$ denote by $\mathcal{N}\left(\nu_{i}, \rho\right)=\left\{\nu \in \rho\right.$ s.t $\left.\left(\nu, \nu_{i}\right) \in \mathcal{E}\right\}$ the set of neighbors of $\nu_{i}$ in the cell $\rho ; \pi$ is an AEP of $\mathcal{G}$ if, for each $i, j \in\{1,2, \ldots, r\}$, with $i \neq j$, there exists an integer $d_{i j}$ such that $\left|\mathcal{N}\left(\nu, \rho_{j}\right)\right|=d_{i j}$ for all $\nu \in \rho_{i}$

\subsection{Problem statement}

Consider $N$ dynamical systems of the form

$$
\begin{aligned}
\dot{x}_{i} & =f_{i}\left(x_{i}\right)+g_{i}\left(x_{i}\right) u_{i} \\
y_{i} & =h_{i}\left(x_{i}\right)
\end{aligned}
$$

with $x_{i} \in \mathbb{R}^{n}, y_{i}, u_{i} \in \mathbb{R}$ for all $i=1, \ldots, N$ and all vector fields and mappings being locally Lipschitz. Roughly speaking, each dynamical system is driven by a fixed linear combination of the outputs of neighbour agents defined by the so-called communication graph $\mathcal{G}=\{\mathcal{V}, \mathcal{E}\}$ (Isidori (2017)). More in details, each node $\nu_{i} \in \mathcal{V}$ is a dynamical system (also referred to as agent) of the form (4); edges of $\mathcal{G}$ specify the interconnections among all agents through the coupling $u_{i}=-\kappa \sum_{\nu_{j} \in \mathcal{N}_{i}}\left(y_{i}-y_{j}\right)$ with $\kappa>0$ being referred to as the strength of the interconnection.

For the sake of compactness and without loss of generality, assuming the Laplacian of the form (1), we shall partition states, outputs and couplings as $\mathbf{x}=$ $\operatorname{col}\left(\mathbf{x}_{1}, \ldots, \mathbf{x}_{\mu}, \mathbf{x}_{\delta}\right) \in \mathbb{R}^{n N}$ with $\mathbf{x}_{i}=\operatorname{col}\left(\left\{x_{j}\right\}_{\nu_{j} \in \mathcal{H}_{i}}\right) \in$ $\mathbb{R}^{n h_{i}}$ for $i=1, \ldots, \mu$ and $\mathbf{x}_{\delta}=\operatorname{col}\left(\left\{x_{j}\right\}_{\nu_{j} \in \mathcal{C}}\right) \in \mathbb{R}^{n \delta}$. In a similar manner, we set $\mathbf{y}=\operatorname{col}\left(\mathbf{y}_{1}, \ldots, \mathbf{y}_{\mu}, \mathbf{y}_{\delta}\right) \in \mathbb{R}^{N}$ and $\mathbf{u}=\operatorname{col}\left(\mathbf{u}_{1}, \ldots, \mathbf{u}_{\mu}, \mathbf{u}_{\delta}\right) \in \mathbb{R}^{N}$.

In this context, we are interested in characterizing the behavior the network induces over each agent (4) under no restriction on the graph properties. Namely, we investigate the evolutions of each node arising from the output-based diffusively interconnection $\mathbf{u}=-\kappa \mathcal{L} \mathbf{y}$ defined by the Laplacian of the graph $\mathcal{G}$.

Complete results for this problem have been provided only for the case of scalar integrators. However, when all nodes are nonlinear heterogeneous agents, only partial results are available and mostly restricted to the case of $\mathcal{G}$ being a directed or undirected but strongly connected graph. Among these, when (4) possesses relative degree $r_{i}=1$, the work in Panteley and Loría (2017) shows that a new (mean-field) behavior is rising and directly affects the evolutions of all agents. However, what about the effect of the network under a general graph $\mathcal{G}$ ?

In the following, the case of scalar integrator dynamics is recalled as a paradigm of the general scenario under investigation.

\subsection{The case of scalar integrators and multi-consensus}

When associating to each node $\nu_{i} \in \mathcal{V}$ a dynamical scalar-integrator system, the Laplacian $\mathcal{L}$ completely governs the behaviors of the overall network (OlfatiSaber et al. (2007); Li et al. (2010); Monaco and Ricciardi Celsi (2019)). Let each agent dynamics (4) specify as

$$
\dot{x}_{i}=u_{i}
$$


with $x_{i} \in \mathbb{R}$ and trivially $y_{i}=x_{i}$. Setting $\mathbf{x}=$ $\operatorname{col}\left(\mathbf{x}_{1}, \ldots, \mathbf{x}_{\mu}, \mathbf{x}_{\delta}\right) \in \mathbb{R}^{N}$ and $\mathbf{u}=-\mathcal{L} \mathbf{x}$, one gets that the networked system is described by

$$
\dot{\mathbf{x}}=-\mathcal{L} \mathbf{x}
$$

As proved in Monaco and Ricciardi Celsi (2019), for all initial conditions $\mathbf{x}(0) \in \mathbb{R}^{N}$, as $t \rightarrow \infty$, agents cluster and the network reaches the multi-consensus according to the almost equitable partition

$$
\pi=\left\{\mathcal{H}_{1}, \ldots, \mathcal{H}_{\mu}, \mathcal{C}_{\mu+1}, \ldots, \mathcal{C}_{\mu+p}\right\}
$$

associated to $\mathcal{G}$ with $\mathcal{C}=\cup_{i=1}^{p} \mathcal{C}_{\mu+i}$. Accordingly, denoting $c_{\ell}=\left|\mathcal{C}_{\mu+\ell}\right|$ for $\ell=1, \ldots, p$ and regrouping nodes in $\mathcal{C}$ in such a way that $\mathbf{x}_{\delta}=\operatorname{col}\left(\mathbf{x}_{\delta_{1}}, \ldots, \mathbf{x}_{\delta_{p}}\right)$ with $\mathbf{x}_{\delta_{\ell}}=\operatorname{col}\left(\left\{x_{j}\right\}_{\nu_{j} \in \mathcal{C}_{\mu+\ell}}\right)$, the following result is recalled.

Theorem 2.1 (Monaco and Ricciardi Celsi (2019)) Consider the multi-agent system (5) with communication graph $\mathcal{G}$ and Laplacian (1). Then, nodes belong to the the same cell $\mathcal{C}_{\mu+\ell} \subseteq \mathcal{C}$ of the $A E P(6)$ if and only if they share the same component of the vector $\gamma^{i}$ in (2). In addition, as $t \rightarrow \infty$ nodes within the same cell of the $A E P$ (6) converge to the same consensus steady-state; that is, rewriting the vectors $\gamma^{i} \in \mathbb{R}^{\delta}$ in (2)

$$
\gamma^{i}=\left(\gamma_{1}^{i} \mathbb{1}_{c_{1}}^{\top} \ldots \gamma_{p}^{i} \mathbb{1}_{c_{p}}^{\top}\right)
$$

(1) for all reaches $\mathcal{H}_{i}$ with $i=1, \ldots, \mu$ and $v_{i}^{\top}$ as in (3)

$$
\mathbf{x}_{i}(t) \rightarrow x_{s, i} \mathbb{1}_{h_{i}}, \quad x_{s, i}=v_{i}^{\top} \mathbf{x}_{i}(0)
$$

(2) for all cells $\mathcal{C}_{\mu+\ell} \subseteq \mathcal{C}$ with $\ell=1, \ldots, p$

$$
\mathbf{x}_{\delta_{\ell}}(t) \rightarrow x_{s, \delta_{\ell}} \mathbb{1}_{c_{\ell}}, \quad x_{s, \delta_{\ell}}=\sum_{i=1}^{\mu} \gamma_{\ell}^{i} x_{s, i}
$$

The network asymptotic behavior clusters: all agents from the same exclusive subgraph (that is $\nu_{j} \in \mathcal{H}_{i}$ ) converge asymptotically to the same consensus value being a mean of the initial states of the corresponding roots; agents in the common part (i.e., $\nu_{j} \in \mathcal{C}$ ) converge to different consensuses being a convex combination of the ones over the reaches.

\section{The main result}

Based on the arguments above, we investigate the socalled topology containment (Liu et al. (2012)) induced by the network over the agents and the possibility of defining a suitably defined set of new dynamics which are paradigmatic of the network collective behavior feeding all agents. In particular, resorting to the results in Panteley and Loría (2017); Lee and Shim (2020), we investigate the structure of the so-called emergent and meanfield dynamics associated to the network and affecting the agents evolutions. In this, sense, based on the AEP (6) of the graph, we show that $\mu+p$ network behaviors emerge which describe the influence of the network connection of the dynamics and force nodes to cluster into $\mu$ independent groups plus further $p$ groups which are influenced by all others. Accordingly, we shall define a multi-consensus error representing the offset among the behavior of each dynamical unit with respect to the network dynamics. Exploiting the partition induced by the Laplacian (1), the agglomerate dynamics reads

$$
\begin{aligned}
\dot{\mathbf{x}}_{i} & =\mathbf{f}_{i}\left(\mathbf{x}_{i}\right)+\mathbf{g}_{i}\left(\mathbf{x}_{i}\right) \mathbf{u}_{i} \\
\dot{\mathbf{x}}_{\delta} & =\mathbf{f}_{\delta}\left(\mathbf{x}_{\delta}\right)+\mathbf{g}_{\delta}\left(\mathbf{x}_{\delta}\right) \mathbf{u}_{\delta} \\
\mathbf{y}_{i} & =\mathbf{h}_{i}\left(\mathbf{x}_{i}\right) \\
\mathbf{y}_{\delta} & =\mathbf{h}_{\delta}\left(\mathbf{x}_{\delta}\right)
\end{aligned}
$$

for $i=1, \ldots, \mu$ and

$$
\begin{aligned}
& \mathbf{f}_{i}(\cdot)=\operatorname{col}\left(\left\{f_{j}(\cdot)\right\}_{\nu_{j} \in \mathcal{H}_{i}}\right), \quad \mathbf{g}_{i}(\cdot)=\operatorname{diag}\left(\left\{g_{j}(\cdot)\right\}_{\nu_{j} \in \mathcal{H}_{i}}\right) \\
& \mathbf{f}_{\delta}(\cdot)=\operatorname{col}\left(\left\{f_{j}(\cdot)\right\}_{\nu_{j} \in \mathcal{C}}\right), \quad \mathbf{g}_{\delta}(\cdot)=\operatorname{diag}\left(\left\{g_{j}(\cdot)\right\}_{\nu_{j} \in \mathcal{C}}\right) \\
& \mathbf{h}_{i}(\cdot)=\operatorname{col}\left(\left\{h_{j}(\cdot)\right\}_{\nu_{j} \in \mathcal{H}_{i}}\right), \quad \mathbf{h}_{\delta}(\cdot)=\operatorname{col}\left(\left\{h_{j}(\cdot)\right\}_{\nu_{j} \in \mathcal{C}}\right) .
\end{aligned}
$$

By taking into account the general form (1) and for $i=$ $1, \ldots, \mu$, the diffusely coupling term $\mathbf{u}=-\kappa \mathcal{L} \mathbf{y}$ reads

$$
\begin{aligned}
\mathbf{u}_{i} & =-\kappa \mathcal{L}_{i} \mathbf{y}_{i} \\
\mathbf{u}_{\delta} & =-\kappa\left(\sum_{j=1}^{\mu} \mathcal{M}_{j} \mathbf{y}_{j}+\mathcal{M} \mathbf{y}_{\delta}\right) .
\end{aligned}
$$

Plugging (9) into (8), the network dynamics is

$$
\begin{aligned}
\dot{\mathbf{x}}_{i}= & \mathbf{f}_{i}\left(\mathbf{x}_{i}\right)-\kappa \mathbf{g}_{i}\left(\mathbf{x}_{i}\right) \mathcal{L}_{i} \mathbf{h}_{i}\left(\mathbf{x}_{i}\right) \\
\dot{\mathbf{x}}_{\delta}= & \mathbf{f}_{\delta}\left(\mathbf{x}_{\delta}\right)-\kappa \mathbf{g}_{\delta}\left(\mathbf{x}_{\delta}\right) \mathcal{M} \mathbf{h}_{\delta}\left(\mathbf{x}_{\delta}\right) \\
& -\kappa \mathbf{g}_{\delta}\left(\mathbf{x}_{\delta}\right) \sum_{j=1}^{\mu} \mathcal{M}_{j} \mathbf{h}_{j}\left(\mathbf{x}_{j}\right)
\end{aligned}
$$

with $i=1, \ldots, \mu$. The above equations underline that the network exhibits a cascade form: for $i=1, \ldots, \mu$, all agents corresponding to a node $\nu_{j} \in \mathcal{H}_{i}$ evolve according to the subgraph associated to $\mathcal{H}_{i}$ and independently of the dynamics in the other reaches and common; the agents belonging to $\mathcal{C}$ a-priori evolve under the influence of all units of the graph. It is intuitively understood that the network is partitioned into at least $\mu+1$ subnetworks composed of $\mu$-independent networks and a further one associated to $\mathcal{C}$. The structure of (10) highlights the existence of $\mu$ mean-field independent dynamics which can be computed exploiting the result in Panteley and Loría (2017) as presented in the next section. 


\subsection{The exclusive mean-field dynamics}

Proposition 3.1 Consider the nonlinear dynamical agents (4) under a communication graph with Laplacian $\mathcal{L}$ of the form (1). Then, the network dynamics (10) exhibits $\mu \geq 1$ independent mean-field dynamics (referred to as exclusive mean-field dynamics) of the form

$$
\begin{aligned}
\dot{\mathbf{x}}_{s, i} & =\mathbf{f}_{s, i}\left(\mathbf{x}_{s, i}\right)+\tilde{\mathbf{f}}_{s, i}\left(\mathbf{x}_{s, i}, \mathbf{e}_{i}\right)-\kappa \tilde{\mathbf{g}}_{s, i}\left(\mathbf{x}_{s, i}, \mathbf{e}_{i}\right) \mathcal{L}_{i} \varepsilon_{i} \\
\mathbf{y}_{s, i} & =\mathbf{h}_{s, i}\left(\mathbf{x}_{s, i}\right)+\tilde{\mathbf{h}}_{s, i}\left(\mathbf{x}_{s, i}, \mathbf{e}_{i}\right)
\end{aligned}
$$

with exclusive mean-field unit and consensus error as

$$
\begin{aligned}
\mathbf{x}_{s, i} & =\left(v_{i}^{\top} \otimes I_{n}\right) \mathbf{x}_{i} \\
\mathbf{e}_{i} & =\mathbf{x}_{i}-\left(\mathbb{1}_{h_{i}} \otimes I_{n}\right) \mathbf{x}_{s, i} \\
\boldsymbol{\varepsilon}_{i} & =\mathbf{y}_{i}-\mathbb{1}_{h_{i}} \mathbf{y}_{s, i}
\end{aligned}
$$

for $i=1, \ldots, \mu, h_{0}=0$ and

$$
\begin{aligned}
\mathbf{f}_{s, i}\left(\mathbf{x}_{s, i}\right) & =\left(v_{i}^{\top} \otimes I_{n}\right) \mathbf{f}_{i}\left(\left(\mathbb{1}_{h_{i}} \otimes I_{n}\right) \mathbf{x}_{s, i}\right) \\
\mathbf{h}_{s, i}\left(\mathbf{x}_{s, i}\right) & =v_{i}^{\top} \mathbf{h}_{i}\left(\left(\mathbb{1}_{h_{i}} \otimes I_{n}\right) \mathbf{x}_{s, i}\right) \\
\tilde{\mathbf{f}}_{s, i}\left(\mathbf{x}_{s, i}, \mathbf{e}_{i}\right) & =\left(v_{i}^{\top} \otimes I_{n}\right) \mathbf{f}_{i}\left(\mathbf{e}_{i}+\left(\mathbb{1}_{h_{i}} \otimes I_{n}\right) \mathbf{x}_{s, i}\right)-\mathbf{f}_{s, i}\left(\mathbf{x}_{s, i}\right) \\
\tilde{\mathbf{g}}_{s, i}\left(\mathbf{x}_{s, i}, \mathbf{e}_{i}\right) & =\left(v_{i}^{\top} \otimes I_{n}\right) \mathbf{g}_{i}\left(\mathbf{e}_{i}+\left(\mathbb{1}_{h_{i}} \otimes I_{n}\right) \mathbf{x}_{s, i}\right) \\
\tilde{\mathbf{h}}_{s, i}\left(\mathbf{x}_{s, i}, \mathbf{e}_{i}\right) & =v_{i}^{\top} \mathbf{h}_{i}\left(\mathbf{e}_{i}+\left(\mathbb{1}_{h_{i}} \otimes I_{n}\right) \mathbf{x}_{s, i}\right)-\mathbf{h}_{s, i}\left(\mathbf{x}_{s, i}\right) .
\end{aligned}
$$

In addition, the consensus error over $\mathcal{C}$ reads

$$
\mathbf{e}_{\delta}=\mathbf{x}_{\delta}-\sum_{i=1}^{\mu}\left(\gamma^{i} \otimes I_{n}\right) \mathbf{x}_{s, i}
$$

Proof. The proof extends the one in Panteley and Loría (2017); Lee and Shim (2020) by computing a suitable network transformation. To this end, consider the Laplacian of the form (1) with the spectral properties underlined in Section 2.1. Then, one can introduce the matrix

$$
Z=\left(\begin{array}{llll}
z_{1} & \ldots & z_{\mu} & Z_{r}
\end{array}\right)
$$

with $Z_{r}$ containing the eigenvectors associated to all other $N-\mu$ non-zero eigenvalues of (1) and, consequently

$$
V^{\top}=Z^{-1}=\left(\begin{array}{c}
\tilde{v}_{1}^{\top} \\
\vdots \\
\tilde{v}_{\mu}^{\top} \\
V_{r}^{\top}
\end{array}\right), \quad V_{r}^{\top}=\left(Z_{r}^{\top} Z_{r}\right)^{-1} Z_{r}^{\top}
$$

such that $J_{\mathcal{L}}=V^{\top} \mathcal{L} Z$ is in Jordan form. Starting from this, we introduce the mapping

$$
\mathbf{x}_{s}=\left(\begin{array}{c}
\mathbf{x}_{s, 1} \\
\vdots \\
\mathbf{x}_{s, \mu}
\end{array}\right)=\left(\begin{array}{c}
\tilde{v}_{1}^{\top} \otimes I_{n} \\
\vdots \\
\tilde{v}_{\mu}^{\top} \otimes I_{n}
\end{array}\right) \mathbf{x}=\left(\begin{array}{c}
\left(v_{1}^{\top} \otimes I_{n}\right) \mathbf{x}_{1} \\
\vdots \\
\left(v_{\mu}^{\top} \otimes I_{n}\right) \mathbf{x}_{\mu}
\end{array}\right)
$$

with $x_{s, i} \in \mathbb{R}^{n}$ for $i=1, \ldots, \mu$ denoting the set of meanfields units. Accordingly, we define

$$
\mathbf{e}=\left(Z_{r} \otimes I_{n}\right)\left(V_{r}^{\top} \otimes I_{n}\right) \mathbf{x}=\left(Z_{r} V_{r}^{\top} \otimes I_{n}\right) \mathbf{x} .
$$

Since by construction $Z_{r} V_{r}^{\top}=I_{N}-\sum_{i=1}^{\mu} z_{i} \tilde{v}_{i}^{\top}$ one gets

$$
\mathbf{e}=\left(\left(I_{N}-\sum_{i=1}^{\mu} z_{i} \tilde{v}_{i}^{\top}\right) \otimes I_{n}\right) \mathbf{x}=\mathbf{x}-\sum_{i=1}^{\mu}\left(z_{i} \otimes I_{n}\right) \mathbf{x}_{s, i}
$$

Accordingly, because of the structure of all right eigenvalues of the Laplacian (1), the error e can be partitioned as $\mathbf{e}=\operatorname{col}\left(\mathbf{e}_{1}, \ldots, \mathbf{e}_{\mu}, \mathbf{e}_{\delta}\right)$ with $\mathbf{e}_{i} \in \mathbb{R}^{n h_{i}}$ with $i=1, \ldots, \mu$ and $\mathbf{e}_{\delta} \in \mathbb{R}^{n \delta}$ as in (12b) and (14). Accordingly, by differentiating each $\mathbf{x}_{s, i}$ one gets

$$
\dot{\mathbf{x}}_{s, i}=\left(v_{i}^{\top} \otimes I_{n}\right)\left(\mathbf{f}_{i}\left(\mathbf{x}_{i}\right)-\kappa \mathbf{g}_{i}\left(\mathbf{x}_{i}\right) \mathcal{L}_{i} \mathbf{y}_{i}\right)
$$

Substituting now $\mathbf{x}_{i}=\mathbf{e}_{i}+\left(\mathbb{1}_{h_{i}} \otimes I_{n}\right) \mathbf{x}_{s, i}$ and $\mathbf{y}_{i}=$ $\varepsilon_{i}+\mathbb{1}_{h_{i}} \mathbf{y}_{s, i}$ in the expression below one gets the result exploiting that $\mathcal{L}_{i} \varepsilon_{i}=\mathcal{L}_{i}\left(\mathbf{y}_{i}-\mathbb{1}_{h_{i}} \mathbf{y}_{s, i}\right)=\mathcal{L}_{i} \mathbf{y}_{i}$.

Each exclusive mean-field unit is uniquely associated to the exclusive reach $\mathcal{H}_{i}$ and corresponds to a weighted average of the states of all agents in the same subnetwork $\mathcal{H}_{i}$. Correspondingly, the multi-consensus error $\mathbf{e}_{i}=$ $\mathbf{x}_{i}-\left(\mathbb{1}_{h_{i}} \otimes I_{n}\right) \mathbf{x}_{s, i}$ defines the behavior of each agent in $\mathcal{H}_{i}$ with respect to the exclusive mean-field unit $\mathbf{x}_{s, i}$ for all $i=1, \ldots, \mu$. In what follows, we refer to (12c) as the output consensus error over $\mathcal{H}_{i}$ whereas, for $\mathcal{C}$, we set

$$
\boldsymbol{\varepsilon}_{\delta}=\mathbf{y}_{\delta}-\sum_{i=1}^{\mu} \gamma^{i} \mathbf{y}_{s, i}
$$

Based on Proposition 3.1, the dynamics within the reach $\mathcal{H}_{i}$ rewrites in terms of the state consensus error as

$$
\begin{aligned}
\dot{\mathbf{e}}_{i}= & -\kappa \Pi_{i} \tilde{\mathbf{g}}_{i}\left(\mathbf{x}_{s, i}, \mathbf{e}_{i}\right) \mathcal{L}_{i} \varepsilon_{i} \\
& +\Pi_{i} \tilde{\mathbf{f}}_{i}\left(\mathbf{x}_{s, i}, \mathbf{e}_{i}\right)+\Pi_{i} \mathbf{f}_{i}\left(\left(\mathbb{1}_{h_{i}} \otimes I_{n}\right) \mathbf{x}_{s, i}\right) .
\end{aligned}
$$

with $\Pi_{i}=\left(\left(I_{h_{i}}-\mathbb{1}_{h_{i}} v_{i}^{\top}\right) \otimes I_{n}\right)$ and

$$
\begin{aligned}
& \tilde{\mathbf{f}}_{i}\left(\mathbf{x}_{s, i}, \mathbf{e}_{i}\right)=\mathbf{f}_{i}\left(\mathbf{e}_{i}+\left(\mathbb{1}_{h_{i}} \otimes I_{n}\right) \mathbf{x}_{s, i}\right)-\mathbf{f}_{i}\left(\left(\mathbb{1}_{h_{i}} \otimes I_{n}\right) \mathbf{x}_{s, i}\right) \\
& \tilde{\mathbf{g}}_{i}\left(\mathbf{x}_{s, i}, \mathbf{e}_{i}\right)=\mathbf{g}_{i}\left(\mathbf{e}_{i}+\left(\mathbb{1}_{h_{i}} \otimes I_{n}\right) \mathbf{x}_{s, i}\right) .
\end{aligned}
$$


In addition, (12c) defines the output consensus error. When the consensus error is indentically zero (11a) reduces to the so-called emergent-dynamics

$$
\dot{\mathbf{x}}_{e, i}=\mathbf{f}_{s, i}\left(\mathbf{x}_{e, i}\right), \quad \mathbf{y}_{e, i}=\mathbf{h}_{s, i}\left(\mathbf{x}_{e, i}\right)
$$

that is, the consensus dynamics over the reach $\mathcal{H}_{i}$.

\subsection{The common mean-field unit}

In this section, we show that further $p \geq 0$ mean-field dynamics arise within the common as suitable combinations of the exclusive ones in (11a). To this end, exploiting Proposition 2.1 and denoting in (1)

$$
\mathcal{M}_{i}=\left(\begin{array}{c}
\mathcal{M}_{i, 1} \\
\vdots \\
\mathcal{M}_{i, p}
\end{array}\right), \quad \mathcal{M}=\left(\begin{array}{ccc}
\mathcal{M}_{11} & \ldots & \mathcal{M}_{1 p} \\
\ddots & \\
\mathcal{M}_{p 1} & \ldots & \mathcal{M}_{p p}
\end{array}\right)
$$

with $\mathcal{M}_{i, \ell} \in \mathbb{R}^{c_{\ell} \times h_{i}}, \mathcal{M}_{\ell_{1} \ell_{2}} \in \mathbb{R}^{c_{\ell_{1}} \times c_{\ell_{2}}}$, nodes in $\mathcal{C}$ are further regrouped so that $(8 \mathrm{~b})$ gets the form

$$
\begin{aligned}
\dot{\mathbf{x}}_{\delta_{\ell}} & =\mathbf{f}_{\delta_{\ell}}\left(\mathbf{x}_{\delta_{\ell}}\right)-\kappa \mathbf{g}_{\delta_{\ell}}\left(\mathbf{x}_{\delta_{\ell}}\right)\left(\sum_{i=1}^{\mu} \mathcal{M}_{i, \ell} \mathbf{y}_{i}+\sum_{q=1}^{p} \mathcal{M}_{\ell q} \mathbf{y}_{q}\right) \\
\mathbf{y}_{\delta_{\ell}} & =\mathbf{h}_{\delta_{\ell}}\left(\mathbf{x}_{\delta_{\ell}}\right)
\end{aligned}
$$

for $\ell=1, \ldots, p$ and

$\mathbf{f}_{\delta_{\ell}}(\cdot)=\operatorname{col}\left(\left\{f_{j}(\cdot)\right\}_{\nu_{j} \in \mathcal{C}_{\mu+\ell}}\right), \mathbf{g}_{\delta_{\ell}}(\cdot)=\operatorname{diag}\left(\left\{g_{j}(\cdot)\right\}_{\nu_{j} \in \mathcal{C}_{\mu+\ell}}\right)$

$\mathbf{h}_{\delta_{\ell}}(\cdot)=\operatorname{col}\left(\left\{h_{j}(\cdot)\right\}_{\nu_{j} \in \mathcal{C}_{\mu+\ell}}\right)$.

At this point, the following result can be proved.

Theorem 3.1 Consider a network of nonlinear dynamical agents (4) coupled via (9) for $\kappa \geq 0$ and graph Laplacian of the form (1). Let (6) be the AEP associated to $\mathcal{G}$. Then, nodes in $\mathcal{C}$ cluster into $p \geq 1$ cells (with $p \geq \delta$ ). Nodes in each $\mathcal{C}_{\mu+\ell}$ are driven by a convex combination of the exclusive mean-field dynamics (12a); namely, for $\ell=1, \ldots, p$ nodes in the same cell $\mathcal{C}_{\mu+\ell} \subset \mathcal{C}$ evolve as

$$
\begin{aligned}
\dot{\mathbf{e}}_{\delta_{\ell}}= & \tilde{\mathbf{f}}_{\delta_{\ell}}\left(\mathbf{x}_{s, \delta_{\ell}}, \mathbf{e}_{\delta_{\ell}}\right)+\mathbf{f}_{\delta_{\ell}}\left(\left(\mathbb{1}_{c_{i}} \otimes I_{n}\right) \mathbf{x}_{s, \delta_{\ell}}\right) \\
& -\sum_{i=1}^{\mu}\left(\gamma_{\ell}^{i} \mathbb{1}_{c_{\ell}} \otimes I_{n}\right)\left(\tilde{\mathbf{f}}_{s, i}\left(\mathbf{x}_{s, i}, \mathbf{e}_{s, i}\right)\right. \\
& \left.+\mathbf{f}_{s, i}\left(\mathbf{x}_{s, i}\right)\right)-\kappa \tilde{\mathbf{g}}_{\delta_{\ell}}\left(\mathbf{x}_{s, \delta_{\ell}}, \mathbf{e}_{\delta_{\ell}}\right) \sum_{j=1}^{p} \mathcal{M}_{\ell j} \varepsilon_{\delta_{\ell}} \\
& -\kappa \sum_{i=1}^{\mu}\left(\tilde{\mathbf{g}}_{\delta_{\ell}}\left(\mathbf{x}_{s, \delta_{\ell}}, \mathbf{e}_{\delta_{\ell}}\right) \mathcal{M}_{\ell, i}\right. \\
& \left.-\left(\gamma_{\ell}^{i} \mathbb{1}_{c_{\ell}} \otimes I_{n}\right) \tilde{\mathbf{g}}_{s, i}\left(\mathbf{x}_{s, i}, \mathbf{e}_{i}\right) \mathcal{L}_{i}\right) \varepsilon_{i}
\end{aligned}
$$

with common mean-field unit given by

$$
\mathbf{x}_{s, \delta_{\ell}}=\sum_{i=1}^{\mu} \gamma_{\ell}^{i} \mathbf{x}_{s, i}
$$

and consensus error

$$
\begin{aligned}
& \mathbf{e}_{\delta_{\ell}}=\mathbf{x}_{\delta_{\ell}}-\left(\mathbb{1}_{c_{\ell}} \otimes I_{n}\right) \mathbf{x}_{s, \delta_{\ell}} \\
& \boldsymbol{\varepsilon}_{\delta_{\ell}}=\mathbf{y}_{\delta_{\ell}}-\mathbb{1}_{c_{\ell}} \mathbf{y}_{s, \delta_{\ell}}
\end{aligned}
$$

and

$$
\begin{aligned}
\tilde{\mathbf{f}}_{\delta_{\ell}}\left(\mathbf{x}_{s, \delta_{\ell}}, \mathbf{e}_{\delta_{\ell}}\right)= & \mathbf{f}_{\delta_{\ell}}\left(\mathbf{e}_{\delta_{\ell}}+\left(\mathbb{1}_{c_{i}} \otimes I_{n}\right) \mathbf{x}_{s, \delta_{\ell}}\right) \\
& -\mathbf{f}_{\delta_{\ell}}\left(\left(\mathbb{1}_{c_{i}} \otimes I_{n}\right) \mathbf{x}_{s, \delta_{\ell}}\right) \\
\tilde{\mathbf{g}}_{\delta_{\ell}}\left(\mathbf{x}_{s, \delta_{\ell}}, \mathbf{e}_{\delta_{\ell}}\right)= & \mathbf{g}_{\delta_{\ell}}\left(\mathbf{e}_{\delta_{\ell}}+\left(\mathbb{1}_{c_{i}} \otimes I_{n}\right) \mathbf{x}_{s, \delta_{\ell}}\right) .
\end{aligned}
$$

Proof. The proof follows partitioning the error defined in the proof of Proposition 3.1 according to the subcells $\mathcal{C}_{\mu+\ell} \subseteq \mathcal{C}$; namely, considering the form (7), one gets

$$
\begin{aligned}
\mathbf{e}_{\delta} & =\mathbf{x}_{\delta}-\sum_{i=1}^{\mu}\left(\gamma^{i} \otimes I_{n}\right) \mathbf{x}_{s, i} \\
& =\left(\begin{array}{c}
\mathbf{x}_{\delta_{1}}-\sum_{i=1}^{\mu} \gamma_{1}^{i}\left(\mathbb{1}_{c_{1}} \otimes I_{n}\right) \mathbf{x}_{s, i} \\
\vdots \\
\mathbf{x}_{\delta_{p}}-\sum_{i=1}^{\mu} \gamma_{p}^{i}\left(\mathbb{1}_{c_{p}} \otimes I_{n}\right) \mathbf{x}_{s, i}
\end{array}\right) \\
\boldsymbol{\varepsilon}_{\delta} & =\mathbf{y}_{\delta}-\sum_{i=1}^{\mu} \gamma^{i} \mathbf{y}_{s, i} \\
& =\left(\begin{array}{c}
\mathbf{y}_{\delta_{1}}-\sum_{i=1}^{\mu} \gamma_{1}^{i} \mathbb{1}_{c_{1}} \mathbf{y}_{s, i} \\
\vdots \\
\mathbf{y}_{\delta_{p}}-\sum_{i=1}^{\mu} \gamma_{p}^{i} \mathbb{1}_{c_{p}} \mathbf{y}_{s, i} .
\end{array}\right)
\end{aligned}
$$

Differentiating the agglomerate common conensus error $\mathbf{e}_{\delta}=\mathbf{x}_{\delta}-\sum_{i=1}^{\mu} \gamma^{i} \mathbf{x}_{s, i}$ one gets

$$
\begin{aligned}
\dot{\mathbf{e}}_{\delta}= & \mathbf{f}_{\delta}\left(\mathbf{x}_{\delta}\right)-\kappa \mathbf{g}_{\delta}\left(\mathbf{x}_{\delta}\right) \mathcal{M} \mathbf{y}_{\delta}-\kappa \mathbf{g}_{\delta}\left(\mathbf{x}_{\delta}\right) \sum_{i=1}^{\mu} \mathcal{M}_{i} \mathbf{y}_{i} \\
& -\sum_{i=1}^{\mu}\left(\gamma^{i} \otimes I_{n}\right)\left(\mathbf{f}_{s, i}\left(\mathbf{x}_{s, i}\right)+\tilde{\mathbf{f}}_{s, i}\left(\mathbf{x}_{s, i}, \mathbf{e}_{i}\right)\right. \\
& \left.-\kappa \tilde{\mathbf{g}}_{s, i}\left(\mathbf{x}_{s, i}, \mathbf{e}_{i}\right) \mathcal{L}_{i} \boldsymbol{\varepsilon}_{i}\right) .
\end{aligned}
$$

At this point, because $\sum_{i=1}^{\mu}\left(\mathcal{M} \gamma^{i}+\mathcal{M}_{i} \mathbb{1}_{h_{i}}\right)=0$

$$
\mathcal{M} \mathbf{y}_{\delta}+\sum_{i=1}^{\mu} \mathcal{M}_{i} \mathbf{y}_{i}=\mathcal{M} \varepsilon_{\delta}+\sum_{i=1}^{\mu} \mathcal{M}_{i} \varepsilon_{i}
$$


so that setting $\mathbf{x}_{s}=\operatorname{col}\left(\mathbf{x}_{s, 1}, \ldots, \mathbf{x}_{s, \mu}\right)$ and

$$
\begin{aligned}
& \tilde{\mathbf{f}}_{\delta}\left(\mathbf{x}_{s}, \mathbf{e}_{\delta}\right)=\mathbf{f}_{\delta}\left(\mathbf{e}_{\delta}+\sum_{i=1}^{\mu}\left(\gamma^{i} \otimes I_{n}\right) \mathbf{x}_{s, i}\right) \\
& \tilde{\mathbf{g}}_{\delta}\left(\mathbf{x}_{s}, \mathbf{e}_{\delta}\right)=\mathbf{g}_{\delta}\left(\mathbf{e}_{\delta}+\sum_{i=1}^{\mu}\left(\gamma^{i} \otimes I_{n}\right) \mathbf{x}_{s, i}\right) .
\end{aligned}
$$

one finally obtains for $\Pi_{\delta}^{i}=\gamma^{i} \otimes I_{n}$

$$
\begin{aligned}
\dot{\mathbf{e}}_{\delta}= & -\kappa \tilde{\mathbf{g}}_{\delta}\left(\mathbf{x}_{s}, \mathbf{e}_{\delta}\right) \mathcal{M} \varepsilon_{\delta}-\kappa \sum_{i=1}^{\mu}\left(\tilde{\mathbf{g}}_{\delta}\left(\mathbf{x}_{s}, \mathbf{e}_{\delta}\right) \mathcal{M}_{i}\right. \\
& \left.-\Pi_{\delta}^{i} \tilde{\mathbf{g}}_{s, i}\left(\mathbf{x}_{s, i}, \mathbf{e}_{i}\right) \mathcal{L}_{i}\right) \varepsilon_{i}+\tilde{\mathbf{f}}_{\delta}\left(\mathbf{x}_{s}, \mathbf{e}_{\delta}\right) \\
& -\sum_{i=1}^{\mu} \Pi_{\delta}^{i}\left(\mathbf{f}_{s, i}\left(\mathbf{x}_{s, i}\right)+\tilde{\mathbf{f}}_{s, i}\left(\mathbf{x}_{s, i}, \mathbf{e}_{i}\right)\right) .
\end{aligned}
$$

Rewriting the equations above component-wise for all $\mathbf{e}_{\delta_{\ell}}$ as in (14) and exploiting (7) one gets the result.

Summarizing, the network dynamics (10) rewrites as the composition of an extra group of topology-induced dynamics (the mean-field) and with respect to which all agents evolve over time (through the consensus error dynamics). The common mean-field unit (20) represents a combination of the exclusive mean-field units underlying the influence of each exclusive reach over the corresponding cluster of nodes of $\mathcal{C}$. As expected, no independent network behavior emerges within the common.

Remark 3.1 The network model inherits the same block-diagonal structure of the Laplacian. Each coefficient $v_{i}^{s}$ of the left eigenvectors $v_{i}^{\top}$ of $\mathcal{L}_{i}$ weights the influence of the corresponding node in the network behavior. Moreover, the components $\gamma^{i}$ of the right eigenvectors $z_{i}$ weight the influence of the corresponding reach over the common $\mathcal{C}$.

Remark 3.2 It must be noted that properties of the mean-field and emergent dynamics are independent on the coordinate employed to represent each agent.

Remark 3.3 In general and even in the LTI case, the state-consensus error (12b)-(14) is never vanishing due to the direct influence of $\mathbf{x}_{s, i}$ over each error-dynamics (16)-(19) through the vector fields $\Pi_{i} \mathbf{f}_{i}\left(\left(\mathbb{1}_{h_{i}} \otimes I_{n}\right) \mathbf{x}_{s, i}\right)$ and $\mathbf{f}_{\delta_{\ell}}\left(\mathbf{x}_{s, \delta_{\ell}}\right)-\sum_{i=1}^{\mu}\left(\gamma_{\ell}^{i} \mathbb{1}_{c_{\ell}} v_{i}^{\top} \otimes I_{n}\right) \mathbf{f}_{i}\left(\left(\mathbb{1}_{h_{i}} \otimes I_{n}\right) \mathbf{x}_{s, i}\right)$.

From (12b)-(12c) and (21a)-(21b), we observe that state consensus (i.e., e $\equiv 0$ ) does not imply output synchronization (i.e., $\varepsilon \equiv 0$ ) in general.

Convergence analysis to state and output multiconsensus can be carried out applying the same results available in the literature for single-consensus of heterogeneous systems depending on the structure of the agents and the framework (e.g., Qu (2009); Frasca et al. (2018); Panteley and Loría (2017); Lee and Shim (2020) to cite a few). Convergence to synchronization does not depend on the communication topology (i.e., on the graph) but only on the agent dynamics.

With these premises and for avoiding redundancy, we are not addressing here the convergence analysis to multiconsensus which can be dealt with, depending on the properties of all agents (4), exploiting standard results in the literature for single consensus.

\section{An example: networks of gravity pendula}

In the simulations we consider a network of $N=7$ agents with communication graph $\mathcal{G}$ with Laplacian

$$
\mathcal{L}=\left(\begin{array}{ccccccc}
2 & -1 & -1 & 0 & 0 & 0 & 0 \\
-1 & 2 & -1 & 0 & 0 & 0 & 0 \\
0 & -1 & 1 & 0 & 0 & 0 & 0 \\
0 & 0 & 0 & 1 & -1 & 0 & 0 \\
0 & 0 & 0 & -1 & 1 & 0 & 0 \\
-1 & 0 & 0 & -1 & 0 & 2 & 0 \\
0 & 0 & -1 & 0 & -1 & -1 & 3
\end{array}\right)
$$

possessing two zero eigenvalues with corresponding left and right eigenvectors

$$
\begin{array}{llll}
v_{1}^{\top}=\left(\begin{array}{lllll}
\frac{1}{6} & \frac{1}{3} & \frac{1}{2} & \mathbf{0}^{\top} & \mathbf{0}^{\top}
\end{array}\right), & v_{2}^{\top}=\left(\begin{array}{lllll}
\mathbf{0}^{\top} & \frac{1}{2} & \frac{1}{2} & \mathbf{0}^{\top}
\end{array}\right) \\
z_{1}^{\top}=\left(\begin{array}{llllll}
1 & 1 & 1 & \mathbf{0}^{\top} & \frac{1}{2} & \frac{1}{2}
\end{array}\right), & z_{2}^{\top}=\left(\begin{array}{lllll}
\mathbf{0}^{\top} & 1 & 1 & \frac{1}{2} & \frac{1}{2}
\end{array}\right) .
\end{array}
$$

Accordingly, nodes can be partitioned as $\mathcal{H}_{1}=$ $\left\{\nu_{1}, \nu_{2}, \nu_{3}\right\}, \mathcal{H}_{2}=\left\{\nu_{4}, \nu_{5}\right\}$ and $\mathcal{C}=\left\{\nu_{6}, \nu_{7}\right\}$ so that, here, $\mu=2$ with $h_{1}=3, h_{2}=2$ and $\delta=2$. The graph admits the almost equitable partition (6) with $p=1$ and $\rho_{3}=\mathcal{C}$. According to Monaco and Ricciardi Celsi (2019), when dealing with simple integrators $\dot{x}_{i}=u_{i}$ with $i=1, \ldots, 7$, three consensuses are expected (as the number of cells of $\pi$ ) depending only on the initial conditions of nodes in the exclusive reaches: one common value for nodes in the same $\mathcal{H}_{i}(i=1,2)$ and one distinct values for the nodes in $\mathcal{C}$. The digraph (22) induces two main main-field behaviors $\mathbf{x}_{s, i}$ with $i=1,2$ each governing the nodes in $\mathcal{H}_{i}$ and the ones in $\mathcal{C}$.

We consider now the case in which agents are gravitypendula described by the Lagrangian equation

$$
\ddot{q}_{i}=-\frac{g}{l_{i}} \sin q_{i}+\frac{1}{m_{i} l_{i}} u_{i}, \quad y_{i}=\dot{q}_{i}
$$

with $l_{i}>0$ and $m_{i}>0$ for $i=1, \ldots, 7$ and $g=9.81$. Denoting $x_{i}=\left(q_{i} \dot{q}_{i}\right)^{\top}$ and assuming an interconnection 
of the form (9) with Laplacian (22), by Proposition 3.1 two exclusive mean-field units arise

$$
\mathbf{x}_{s, 1}=\frac{1}{6} x_{1}+\frac{1}{3} x_{2}+\frac{1}{2} x_{3}, \quad \mathbf{x}_{s, 2}=\frac{1}{2}\left(x_{4}+x_{6}\right)
$$

with corresponding outputs

$$
\mathbf{y}_{s, 1}=\frac{1}{6} \dot{q}_{1}+\frac{1}{3} \dot{q}_{2}+\frac{1}{2} \dot{q}_{3}, \quad \mathbf{y}_{s, 2}=\frac{1}{2}\left(\dot{q}_{4}+\dot{q}_{6}\right)
$$

Accordingly, the emergent dynamics is provided by the Lagrangian system

$$
\ddot{\mathbf{q}}_{e, 1}=-g\left(\frac{l_{1}}{6}+\frac{l_{2}}{3}+\frac{l_{3}}{2}\right)^{-1} \sin \mathbf{q}_{e, 1} .
$$

As far as the reach component is concerned, exploiting Theorem 3.1 the corresponding mean-field units and the corresponding outputs are given by

$$
\mathbf{x}_{s, \delta}=\frac{1}{2}\left(\mathbf{x}_{s, 1}+\mathbf{x}_{s, 2}\right), \quad \mathbf{y}_{s, \delta}=\frac{1}{2}\left(\mathbf{y}_{s, 1}+\mathbf{y}_{s, 2}\right) .
$$

One can verify, in general, that unless $m=m_{i}$ and $l=l_{i}$ $(i=1, \ldots, 7)$ the emergent dynamics is not attractive for all agents (i.e., asymptotic state multi-consensus cannot be achieved). Exploiting the result in (Qu (2009)) for single-consensus, by passivity of all agents with storage

$$
S_{i}\left(x_{i}\right)=\frac{1}{2} \dot{q}_{i}^{2}+\frac{g}{l_{i}}\left(1-\cos q_{i}\right)
$$

asymptotic output multi-consensus is guaranteed; namely, as $t \rightarrow \infty$ one gets

$$
\begin{aligned}
\dot{q}_{i} \rightarrow \mathbf{y}_{s, 1} & =\frac{1}{6} \dot{q}_{1}+\frac{1}{3} \dot{q}_{2}+\frac{1}{2} \dot{q}_{3}, \quad i=1,2,3 \\
\dot{q}_{i} \rightarrow \mathbf{y}_{s, 2} & =\frac{1}{2}\left(\dot{q}_{4}+\dot{q}_{6}\right), \quad i=4,5 \\
\dot{q}_{i} \rightarrow \mathbf{y}_{s, \delta} & =\frac{1}{12} \dot{q}_{1}+\frac{1}{6} \dot{q}_{2}+\frac{1}{4} \dot{q}_{3}+\frac{1}{4}\left(\dot{q}_{4}+\dot{q}_{5}\right), \quad i=6,7
\end{aligned}
$$

For completeness, a simple simulation is reported in Figure 1 setting $\kappa=10, m_{i}=i$ and $l_{i}=i+1$ for $i=$ $1, \ldots, 7$. The result highlights that all agents belonging to the same reach of the AEP converge to a shared output trajectory provided by the weighted mean (through (3)) of the outputs of the corresponding agents; nodes in the common converge to a further output consensus dictated by the nodes in the exclusive reach only. As expended, despite output consensus is achieved, the connection fails in guaranteeing state consensus even when all agents have identical masses.

\section{Conclusions}

In this paper, we have characterized the behaviors induced by the network interconnection of heterogeneous nonlinear systems under a fixed but general graph topology. We have emphasized that, as suggested by the single-integrator case, the network induces a specific partition of the nodes which also governs the cluster collective behaviors. Current work is addressing the case of time-varying topology and the possibility of embedding feedback design to change both the agents dynamics and the connecting topology to obey to certain specifications in a unifying framework for different multi-agent control problems such as randez-vous, flocking or formation. A preliminary work in this darection is Cacace et al. (2021) where the case of LTI homogeneous agents has been addressed.

\section{References}

Aeyels, D., De Smet, F., 2010. Emergence and evolution of multiple clusters of attracting agents. Physica D: Nonlinear Phenomena 239, 1026-1037.

Agaev, R., Chebotarev, P., 2005. On the spectra of nonsymmetric Laplacian matrices. Linear Algebra and its Applications 399, 157-168.

Arenas, A., Díaz-Guilera, A., Kurths, J., Moreno, Y., Zhou, C., 2008. Synchronization in complex networks. Physics reports 469, 93-153.

Battilotti, S., Califano, C., 2019. Leader-following consensus for nonlinear agents with measurement feedback. International Journal of Robust and Nonlinear Control 29, 1694-1718.

Cacace, F., Mattioni, M., Monaco, S., Ricciardi Celsi, L., 2021. Topology-induced containment for general linear systems on weakly connected digraphs. Automatica 131, 109734.

Cardoso, D.M., Delorme, C., Rama, P., 2007. Laplacian eigenvectors and eigenvalues and almost equitable partitions. European Journal of Combinatorics $28,665-673$.

Caughman, J.S., Veerman, J., 2006. Kernels of directed graph Laplacians. The Electronic Journal of Combinatorics 13,39 .

Chen, Y., Lü, J., Han, F., Yu, X., 2011. On the cluster consensus of discrete-time multi-agent systems. Systems \& Control Letters 60, 517-523.

Chen, Y., Lu, J., Yu, X., Hill, D.J., 2013. Multi-agent systems with dynamical topologies: Consensus and applications. IEEE Circuits and Systems Magazine $13,21-34$.

Cristofaro, A., Mattioni, M., 2021. Hybrid consensus for multi-agent systems with time-driven jumps. Nonlinear Analysis: Hybrid Systems 43, 101113.

Cui, R., Ren, B., Ge, S.S., 2012. Synchronised tracking control of multi-agent system with high-order dynamics. IET Control Theory \& Applications 6, 603-614.

DeLellis, P., Falanga, G., Garofalo, F., Iudice, F.L., 2018. Topology control of networks of proximity kuramoto oscillators, in: 2018 European Control Conference (ECC), IEEE. pp. 2194-2199.

Dimarogonas, D.V., Frazzoli, E., Johansson, K.H., 2012. 

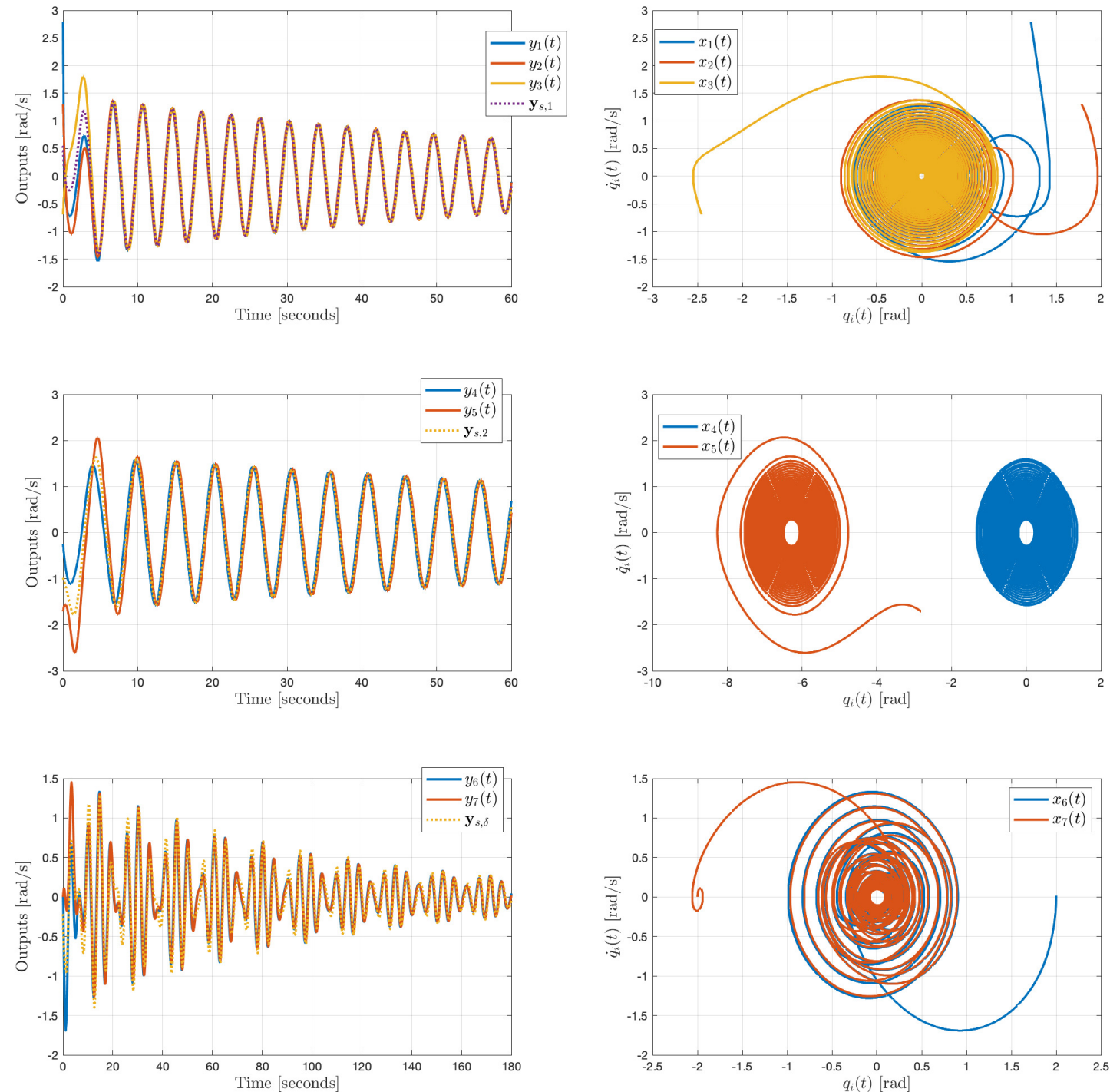

Fig. 1. Network of gravity pendula

Distributed event-triggered control for multi-agent systems. IEEE Transactions on Automatic Control 57, 1291-1297.

Egerstedt, M., Martini, S., Cao, M., Camlibel, K., Bicchi, A., 2012. Interacting with networks: How does structure relate to controllability in single-leader, consensus networks? IEEE Control Systems Magazine $32,66-73$.

Frasca, M., Gambuzza, L.V., Buscarino, A., Fortuna, L., 2018. Synchronization in Networks of Nonlinear Circuits: Essential Topics with MATLABßR Code.
Springer.

Gambuzza, L.V., Frasca, M., 2020. Distributed control of multi-consensus. IEEE Transactions on Automatic Control , 1-1doi:10.1109/TAC.2020.3006820.

Isidori, A., 2017. Lectures in feedback design for multivariable systems. volume 3 of Advanced Textbooks in Control and Signal Processing. Springer.

Jadbabaie, A., Lin, J., Morse, A.S., 2003. Coordination of groups of mobile autonomous agents using nearest neighbor rules. Departmental Papers (ESE), 29.

Lee, J.G., Shim, H., 2020. A tool for analysis and synthe- 
sis of heterogeneous multi-agent systems under rankdeficient coupling. Automatica 117, 108952.

Li, Z., Duan, Z., Chen, G., Huang, L., 2010. Consensus of multiagent systems and synchronization of complex networks: A unified viewpoint. IEEE Trans. on Circuits and Systems I: Regular Papers 57, 213-224.

Liu, H., Xie, G., Wang, L., 2012. Containment of linear multi-agent systems under general interaction topologies. Systems \& Control Letters 61, 528-534.

Mattioni, M., 2020. On multiconsensus of multi-agent systems under aperiodic and asynchronous sampling. IEEE Control Systems Letters 4, 839-844.

Monaco, S., Ricciardi Celsi, L., 2019. On multiconsensus and almost equitable graph partitions. Automatica $103,53-61$.

Monshizadeh, N., Zhang, S., Camlibel, M.K., 2015. Disturbance decoupling problem for multi-agent systems: A graph topological approach. Systems \& Control Letters 76, 35-41.

Moreau, L., 2005. Stability of multiagent systems with time-dependent communication links. IEEE Transactions on automatic control 50, 169-182.

Olfati-Saber, R., Fax, J.A., Murray, R.M., 2007. Consensus and cooperation in networked multi-agent systems. Proceedings of the IEEE 95, 215-233.

Panteley, E., Loría, A., 2017. Synchronization and dynamic consensus of heterogeneous networked systems. IEEE Transactions on Automatic Control 62, 37583773.

Pasqualetti, F., Dörfler, F., Bullo, F., 2013. Attack detection and identification in cyber-physical systems. IEEE Transactions on Automatic Control 58, 27152729.

Pietrabissa, A., Suraci, V., 2017. Wardrop equilibrium on time-varying graphs. Automatica 84, 159-165.

Qu, Z., 2009. Cooperative control of dynamical systems: applications to autonomous vehicles. Springer Science \& Business Media.

Ren, W., Cao, Y., 2010. Distributed coordination of multi-agent networks: emergent problems, models, and issues. Springer Science \& Business Media.

Sun, Y.G., Wang, L., 2009. Consensus problems in networks of agents with double-integrator dynamics and time-varying delays. International Journal of Control 82, 1937-1945.

Trumpf, J., Trentelman, H.L., 2018. Controllability and stabilizability of networks of linear systems. IEEE Transactions on Automatic Control .

Wang, X., Lu, J., 2019. Collective behaviors through social interactions in bird flocks. IEEE Circuits and Systems Magazine 19, 6-22.

Wen, G., Duan, Z., Yu, W., Chen, G., 2013. Consensus of multi-agent systems with nonlinear dynamics and sampled-data information: a delayed-input approach. International Journal of Robust and Nonlinear Control 23, 602-619.

Xiao, F., Wang, L., 2006. Consensus behavior of agents in networked systems under general communication topologies, in: 2006 IEEE Conference on Com- puter Aided Control System Design, 2006 IEEE International Conference on Control Applications, 2006 IEEE International Symposium on Intelligent Control, IEEE. pp. 862-867.

Zhan, J., Li, X., 2013. Consensus of sampled-data multiagent networking systems via model predictive control. Automatica 49, 2502-2507. 